\title{
Sexual Acquisition and Transmission of HIV Cooperative Agreement Program (SATHCAP), July 2009
}

\section{Introduction}

\author{
Wilson Compton, Jacques Normand, and Elizabeth Lambert
}

In 2003, the National Institute on Drug Abuse (NIDA) of the National Institutes of Health invited collaborative research proposals on the sexual transmission of HIV and other infectious diseases among drug-using and non-drug-using populations. The outcome of this effort was the cooperative agreement program called the "Sexual Acquisition and Transmission of HIV Cooperative Agreement Program" or SATHCAP. SATHCAP was designed for multidisciplinary epidemiologic research on the behavioral, biological, and environmental processes involved in the sexual transmission of HIV and other sexually transmitted infections/diseases (STD) among drug users and to non-drug-using populations.

The origins of this initiative actually began long before 2003, as the then oftencited (and recently revised upward) estimate of incident HIV in the USA had, after peaking at 160,000 in the mid-1980s, held steady at approximately 40,000 new infections since 1990. It was that persistent annual rate of 40,000 new infections that called attention to the gaps in our knowledge of the risk factors for new infections and, as importantly, of how to improve our HIV prevention and intervention strategies to reduce the number of incident cases. In addition, NIDA-supported researchers $^{1,2}$ had recently reported that, among injecting drug users (IDUs), HIV seroconversion was strongly associated with sexual risk behaviors, that the strongest predictor of HIV seroconversion among male IDUs was having sex with men, that among female IDUs, the strongest predictor was trading sex for money, and that the proportion of HIV infections attributable to sexual risks among IDU was unexpectedly large, though not larger than that attributable to injection-drug-use risks. These findings underscored reports by others ${ }^{3}$ on the increasing complexity of HIV, the multiplicity of risks for this disease, and the need for better understanding of risk factors for sexual HIV transmission among drug users. They also suggested that traditional schemes for classifying HIV risk, that is by using single, exclusionary exposure categories, may have, at best, oversimplified our knowledge or, at worst, impeded our ability for public health action to slow and avert new infections.

NIDA recognized then that a multidisciplinary perspective was needed to understand how, why, and among whom the epidemic of HIV/AIDS was changing. ${ }^{4}$ This recognition, coupled with the need for broad-scale advancements in more effective and sustainable HIV interventions to reduce, prevent, and/or eliminate new

Compton and Lambert are with the Division of Epidemiology, Services, and Prevention Research, National Institute on Drug Abuse, Bethesda, MD, USA; Normand is with the AIDS Research Program, National Institute on Drug Abuse, Bethesda, MD, USA.

Correspondence: Elizabeth Lambert, Division of Epidemiology, Services, and Prevention Research, National Institute on Drug Abuse, 6001 Executive Blvd., Bethesda, MD 20892, USA. (E-mail: elambert@nida.nih.gov) 
infections, became the springboard to the SATHCAP initiative. Researchers from multiple disciplines, including the behavioral, biological, and biomedical sciences, sociology, and mathematics, were invited to submit collaborative research applications that addressed the changing epidemiology of HIV/AIDS among drug users and from drug users to non-drug-using populations and that would help us identify new opportunities to improve the effectiveness of HIV prevention interventions.

We are pleased to introduce this Special Issue of the Journal of Urban Health, with findings from each of the four SATHCAP research sites as well as from the RAND Coordinating Center. The four research sites include three large and diverse geographic areas in the USA (Chicago, IL; Los Angeles, CA; and Raleigh Durham, NC) and an international site, St Petersburg, Russia. Each paper provides a unique, yet complementary, perspective on the changing epidemiology of HIV/AIDS. The SATHCAP investigators decided early on to focus at least one of their two papers for this Special Issue on the cross-site theme of "bridging" in the sexual transmission of HIV, that is, on how the connections among and between higher risk groups (drug users, men who have sex with men (MSM)) and lower risk groups in the general population (non-drug users, sexual partners of drug users, and female partners of men who have sex with men and women) are continually changing, and what the consequences of such change may be for the general diffusion of HIV (as well as other STDs).

We will briefly mention the papers that make up this Special Issue here, in part to entice the reader's interest to read on but more to acknowledge the superb efforts by the authors and their SATHCAP teams who, quite literally, have dedicated themselves to this project over the past 6 years. These papers touch upon the complexity of issues and challenges involved in SATHCAP, yet in actuality, they represent the mere "tip of the iceberg." To be sure, SATHCAP was, and is, a complex and multidisciplinary project, and we expect its data will continue to be a source for further study and publications in the years to come.

The overview paper, by Iguchi et al., is largely devoted to the design of SATHCAP, with special attention given to its sampling strategy, that is, respondentdriven sampling (RDS). As the paper describes, SATHCAP discovered that RDS has strengths and, somewhat unexpectedly, a number of limitations that required adjustments to the sampling plan as time went on. One site, Los Angeles, experienced a problem with RDS known as homophily, where participants recruit others just like themselves to such an extent that the final sample represents a limited segment, albeit an important one, of the actual population of interest. Nonetheless, despite the challenges of RDS, as Iguchi et al. discuss, there were a number of findings—as well as methodological lessons and new research questions-arising from SATHCAP that will interest the reader and potentially inspire future research on the changing epidemiology of HIV/AIDS.

Two papers in the Special Issue are by Zule et al., of the Raleigh/Durham research site, which included both urban and nearby rural counties. One paper examines the extent to which men who have sex with men and women may serve as a bridge between higher and lower risk groups, leading to potential HIV diffusion to the general population. The other focuses on the correlates and predictors of transactional sex, reported by $30 \%$ of women and $25 \%$ of men in the study sample about at least one of their last three partners. As the findings suggest, transactional sex for money, drugs, food, or other items, has immediate and long-term implications for the potential spread of HIV in urban areas as well as to remote and less populated rural counties. 
The papers from the Los Angeles site are by Shoptaw et al. and Gorbach et al., respectively. In the first, Dr Shoptaw and colleagues explore differences in "internalized homonegativity" among the young MSM and MSM who also have sex with women participating in SATHCAP. They found interesting differences in internalized homonegativity among study participants which correlated with differences in HIV-related drug and sexual risk behaviors, HIV status, and ever having a prior test for HIV, and which have clear implications for public health efforts to focus HIV testing and prevention efforts on those with greatest need. The paper by Dr. Gorbach and colleagues returns to the cross-site theme of bridging by analyzing the potential contribution of bisexual men who use drugs to the diffusion of HIV and other STDs in Los Angeles.

The two papers by the Chicago site address the theme of bridging, in one, and the characteristics and risk practices of bisexual, low income, and drug-using men in Chicago, in the other. We would like to call the reader's attention to this bridging paper, in particular, because it goes beyond an analysis of sexual network linkages between individuals and groups to an analysis of potential bridging communities, using as its analytic units the 77 community areas established by Chicago years ago.

The two papers in the Special Issue from the international research site, St. Petersburg, Russia, are both devoted to the theme of bridging. Dr. Toussova and colleagues present findings from their analysis of the sex partners of 288 non-drug users whose HIV diagnosis was attributed to sexual exposure. Their paper focuses on the potential role of IDU sex partners as bridges of HIV transmission to the St. Petersburg population, with particular attention to age and gender differences and how these may impact the city's expanding epidemic. Dr. Niccolai et al. also address the bridging theme in their paper, which nicely complements that of Dr. Toussova and colleagues. Their analysis examines the extensive bridging partnerships reported by the drug users and non-drug-using partners of drug users recruited into SATHCAP. With the high prevalence of HIV among IDU, the increasing prevalence among nondrug users and the high rate of unsafe sex reported by both drug users and their nondrug-using partners, it appears that the rapid growth of HIV in St. Petersburg and nearby rural provinces will continue with no end in sight.

The papers in this Special Issue from the USA sites suggest that the HIV/AIDS epidemic has matured in the USA. The exponential spread of infection that marked the early phase of the epidemic has been replaced by a pattern of general stability; although HIV prevalence in the USA has increased, it has done so because more people are living longer with the help of new therapies. The Communicable Disease Centre (CDC) recently released a new HIV incidence estimate in the USA of 56,300 infections in 2006, which compares with the long-cited estimate of 40,000 annual new infections in prior years. ${ }^{5}$ The new estimate results from a more accurate way of measuring new infections rather than from an actual increase and, as the CDC notes, a separate trend analysis shows that the annual number of new infections in the USA has remained stable since the late 1990s. This macro-pattern, while generally reassuring, does not preclude localized perturbations, with "flares" of epidemic spread among high-risk subgroups or a rise in an area's endemic transmission rate. By contrast, the papers from the St. Petersburg, Russia site reveal an epidemic at an earlier phase, with new infections spreading at a rapid rate among diverse risk groups, including not only injection drug users but also their heterosexual partners, the non-drug user sex partners of sex partners, and among men who have sex with men. 
SATHCAP addressed a central question about the current epidemiology and transmission dynamics of HIV/AIDS: To what extent does HIV diffuse from groups known to be at high risk, such as drug users and men who have sex with men, to others at lower risk, such as non-drug-using sex partners, or the sex partners of sex partners? In the USA, the answer for now seems to be: not much; and in St. Petersburg, Russia, the answer seems to be: a lot. The high prevalence of HIV-related risk behaviors found among SATHCAP participants in the USA, including the widespread use of different drugs and very high rates of unsafe sex with multiple partners, reminds us that control of this epidemic requires an actual understanding of risk mechanisms and dynamics and not just the monitoring of secular trends. Certainly, the findings for Russia cry out for a major public health response to avert the continuing rapid spread of sexual HIV transmission among drug users as well as from drug users to non-drug-users and to their heterosexual partners.

\section{REFERENCES}

1. Kral AH, Bluthenthal RN, Lorvick J, Gee L, Bacchetti P, Edlin BR. Sexual transmission of HIV-1 among injection drug users in San Francisco, USA: risk-factor analysis. Lancet. 2001; 357(9266): 1397-1401.

2. Strathdee SA, Sherman SG. The role of sexual transmission of HIV infection among injection and non-injection drug users. J Urban Health. 2003; 80(4 suppl 3): iii7-14.

3. Rothenberg RB, Long DM, Sterk CE, Pach A, Potterat JJ, Muth S, Baldwin JA, Trotter RT. The Atlanta urban network study: a blueprint for endemic transmission. AIDS. 2000; 14: 2191-2200.

4. Normand, JL, Lambert, EY, D. Vlahov (eds). Understanding the dynamics of sexual transmission of HIV among drug-using populations: an integration of biological, behavioral, and environmental perspectives. J Urban Health. 2003; 80(4): iii1-iii6.

5. Hall HI, Song R, Rhodes P, Prejean J, An Q, Lee LM, Karon J, Brookmeyer R, Kaplan EH, McKenna MT, Janssen RS, for the HIV Incidence Surveillance Group. Estimation of HIV Incidence in the United States. JAMA. 2008; 300(5): 520. 